\title{
BMJ Open Assessing the quality, efficiency and usefulness of the Western Australian population-based Intellectual Disability Exploring Answers (IDEA) surveillance system: a surveillance system evaluation
}

\author{
Natalie A Strobel (D) , ${ }^{1,2}$ Jenny Bourke, ${ }^{3}$ Helen Leonard (D) , ${ }^{3}$ Alice Richardson (D) , ${ }^{2}$ \\ Karen M Edmond, ${ }^{4}$ Daniel McAullay ${ }^{1}$
}

To cite: Strobel NA, Bourke J, Leonard $\mathrm{H}$, et al. Assessing the quality, efficiency and usefulness of the Western Australian population-based Intellectual Disability Exploring Answers (IDEA) surveillance system: a surveillance system evaluation. BMJ Open 2019;9:e026003. doi:10.1136/ bmjopen-2018-026003

- Prepublication history and additional material for this paper are available online. To view these files, please visit the journal online (http://dx.doi org/10.1136/bmjopen-2018026003).

Received 14 August 2018 Revised 20 August 2019 Accepted 16 September 2019

Check for updates

(c) Author(s) (or their employer(s)) 2019. Re-use permitted under CC BY-NC. No commercial re-use. See rights and permissions. Published by BMJ.

${ }^{1}$ Medical School, University of Western Australia, Crawley, Western Australia, Australia ${ }^{2}$ NCEPH, Australian National University, Canberra, Australian Capital Territory, Australia

${ }^{3}$ Telethon Kids Institute, West

Perth, Western Australia,

Australia

${ }^{4}$ Department Of Women \& Children's Health, King's College London, London, UK

Correspondence to Dr Natalie A Strobel; natalie.strobel@uwa.edu.au

\section{ABSTRACT}

Objectives Our overall aim was to evaluate the Western Australian Intellectual Disability Exploring Answers (IDEA) surveillance system. The primary objective was to evaluate the attributes of the system. The secondary objective was to provide recommendations to data custodians and stakeholders to strengthen the system.

Method The IDEA system was evaluated using process observation, interviews and secondary data analysis of system attributes: usefulness, simplicity, data quality, acceptability, representativeness, timeliness and stability. 2001 US Centers for Disease Control and Prevention guidelines were used.

Results We found that the IDEA system was useful, simple, flexible, acceptable, representative, timely and stable. We compared individuals from the IDEA system $(n=10593)$ with those with cerebral palsy and intellectual disability (ID) $(\mathrm{n}=582)$ from another surveillance system. Of the 582 with cerebral palsy and ID, $501(86.1 \%)$ were in the IDEA system and 81 (13.9\%) were not. In total, 0.7\% of cases (81/10674) with ID were not identified in the IDEA system. There were little differences in cases that were not identified in the IDEA system between Indigenous status, sex and place of residence.

Conclusions The strengths of the IDEA system include having a high data quality resource contributing to national and international data on ID, strong government support and a dedicated management team. Output from studies linking to IDEA data have had major contributions to the international literature about ID. However, limited resources have prevented it from realising its full potential in relation to translational activities. The IDEA system is a valuable resource to address the needs of people living with ID.

\section{INTRODUCTION}

People living with an intellectual disability (ID) have impaired thought processes, learning, communication and remembering, which contribute to their overall intelligence including cognition, language, and may affect motor and social abilities. As a result,

\section{Strengths and limitations of this study}

- The methods used are highly acceptable for evaluating surveillance systems.

- We evaluated the system through process observation, interviews and secondary data analysis.

- We assessed the attributes usefulness, simplicity, data quality, acceptability, representativeness, timeliness and stability.

- We investigated the completeness of the Western Australian Intellectual Disability Exploring Answers system by cross-checking individuals from the mandatory Western Australian Registry of Developmental Anomalies-Cerebral Palsy database with individuals who have intellectual disability.

- We were unable to compare the system attributes positive predictive value and sensitivity due to a lack of primary data.

people with IDs are more likely to suffer from maltreatment as children, ${ }^{1}$ have increased comorbidities, ${ }^{2}$ mental health diagnoses ${ }^{3}$ and often experience stigmatisation and discrimination resulting in poor access to health services ${ }^{4}$ compared with their counterparts who do not have an ID. In addition, with advances in healthcare, many people with an ID now have elderly carers or will outlive their carers. Additional government input for care services previously managed by families will be needed in the years to come. ${ }^{5}$

Internationally, there are few dedicated public health surveillance systems for ID. Many rely on data from existing state and national surveys, administrative datasets, registries or integrated data systems. ${ }^{6-8}$ In Western Australia, the Intellectual Disability Exploring Answers (IDEA) database is a population-based linked data surveillance system, which is internationally recognised 
for its collection of prevalence and incidence data for ID. ${ }^{9}$ The original objective of the surveillance system was to provide high-quality, complete and population-based information on ID in Western Australia. It was anticipated that this information might be used for the following purposes: monitoring trends and investigating changes in the prevalence of ID, overall and in various subgroups; providing an infrastructure for population-based epidemiological and genetic research into the causes and prevention of ID; providing an infrastructure for research into the health status and service needs of children and adults with ID; allowing the identification of population-based subgroups with specific characteristics who might benefit from new scientific advances; evaluating screening programmes for prevention of ID; facilitating planning and providing infrastructure for the evaluation of early intervention and therapy programmes and increasing community and professional knowledge about ID. ${ }^{10}$

The Australian National Disability Insurance Scheme has recently been introduced in Australia and provides funding for supports and services for people living with a significant and permanent disability. ${ }^{11}$ As a result, the Disability Services Commission (DSC; now Department of Communities) will no longer provide services for people with ID in Western Australia and in the long run will not provide data to IDEA. Currently, proof-of-concept efforts are underway to pilot the process for the IDEA to receive data from the Australian Commonwealth government on people living in Western Australia with ID. In addition, awareness of the current strengths and limitations of the IDEA surveillance system could help stakeholders and data custodians better recognise the need and value of this system in light of current policy initiatives. Therefore, an evaluation of the IDEA surveillance system was undertaken to assess the quality, efficiency and usefulness of the system. The primary objective was to systematically and objectively evaluate the attributes of the system. The secondary objective was to provide recommendations to data custodians and stakeholders to strengthen the surveillance system.

\section{METHODS \\ Design}

This evaluation is based on the methods from the 2001 US Centers for Disease Control and Prevention guidelines on evaluation of public health surveillance systems. ${ }^{11}$ We assessed the following system attributes: usefulness (how important is the collection of ID; does it respond to prevention, early detection and evaluation of programmes or improve public health knowledge), simplicity (ease of understanding data processes), flexibility (ability of the system to adapt to changing needs), data quality (is the data complete), acceptability (the willingness of providers to participate in the IDEA system processes), representativeness (are the data generalisable to the wider population), timeliness (speed at which data are provided at all stages) and stability (whether resourcing is sufficient). We did not aim to calculate positive predictive value and sensitivity due to lack of primary data to assess these attributes.

\section{Study setting}

Case ascertainment and eligibility

Cases are ascertained from the DSC through referrals to assess individuals for eligibility to access disability services. For the Department of Education cases are school aged children who are identified as potentially needing additional teaching support in relation to intellectual functioning and who have been assessed to determine the level of educational support required. The online supplementary material provides case eligibility for both DSC and Department of Education.

Eligibility for IDEA has been extended to children $<6$ years of age who are considered 'vulnerable' by the DSC when a developmental assessment indicates a likelihood of ID, although they are too young to have a formal IQ assessment. These children are included in the database but are reconsidered if assessments become available at school age. This represents only approximately $2 \%$ of cases (estimated for birth years 1990-2001). ${ }^{9}$ Children identified through the Department of Education were accepted as having an ID unless there is conflicting evidence from DSC.

\section{Case definition}

A confirmed case from the DSC is (i) an individual with a full $\mathrm{IQ}<70$; (ii) evidence of developmental delay at $<18$ years of age (where evidence is not available but there is no obvious cause for the ID after 18 years of age, it is accepted that the delay was probably present during childhood and the case will be eligible) or (iii) where there is no IQ test score available but the child has a known biomedical cause of ID, such as Down syndrome.

Prior to 2006, confirmed cases from the Department of Education were included if the assigned level of ID was 'mild or moderate' or severe. Subsequent to 2005 and in the absence of availability of information on ID level, cases with an educational need of 4-5 were considered to have an ID. An analysis of the correlation between the previously assigned level of ID and the level of educational need has shown that an educational need score of 4 is correlated with a mild or moderate ID, and an educational need score of 5 with a severe ID. ${ }^{9}$ In 2016 , the level of educational need was replaced with an Individual Disability Allocation, which was rated from 1 (mild ID) to 7 (severe and comorbid ID) and used to estimate level of ID. Further enhancement of data is undertaken by a medical officer, located at DSC, using the four-digit American Association on Mental Retardation (AAMR) system to assign the most appropriate cause of ID to cases ${ }^{12}$ which can be later grouped into broader categories. 
Management of IDEA system

Currently, there is funding provided by the DSC for personnel equivalent to 0.5 FTE and operating costs. Personnel costs cover liaising with departments for data, updating data within the IDEA system, supporting and completing epidemiological studies on ID and responding to requests for data. Operating costs need to cover future fees for data linkage by Department of Health Western Australia. Funds have also provided some support for traditional research outputs such as conference fees and publication costs. However, there has been limited support for work related travel, communication and engagement activities, which have been covered from other sources including a philanthropic donation in 2013. In addition, there is a volunteer community advisory group which consists of researchers, advocates for ID, policy makers and the IDEA system data custodian. The aim of the advisory group is to review and approve projects applying for the use of ID data in their study and to provide support where applicable. Although the advisory committee originally met annually in person, since 2011 communication between members has primarily been through email.

\section{Patient and public involvement}

Patient and public involvement were not completed for the study design or the development of outcome measures. A member of the public and an advocacy organisation for ID were recruited and provided their views on the IDEA system. The results will be disseminated through traditional journal publication, conference presentation and a lay summary, which will be sent to all individuals who participated in the project. We have acknowledged the time stakeholders spent in participating in the study.

\section{Data collection}

For privacy and confidentiality reasons there is a limited number of data variables that are collected as part of the IDEA system (online supplementary table 1). As a population-based data linkage surveillance system these data need to be linked to other Western Australian administrative data collections.

The process of obtaining data for the IDEA system involves data contributions from two Western Australian government departments, the DSC and the Department of Education. Figure 1 provides a flow diagram outlining the process from case ascertainment to finalising the IDEA system updates. Identifiable data on individuals with probable and suspected ID are provided to the Data Linkage Branch, Department of Health Western Australia. These data are de-identified and only linkable through unique codes called root numbers, which are then provided back to the IDEA custodian and to the respective departments. Both departments then provide the IDEA management team with their de-identified datasets and data variables. This process is undertaken to safeguard privacy and confidentiality at all stages and takes approximately 9 months to occur. Once the data are received by the IDEA team duplicates are combined into one record, new records are assessed for eligibility and the system updated (figure 1). This latter process takes approximately 4 months to complete. Updates from DSC and the Education Department were initially undertaken every 2 years. However, there were 4 years between the last two updates (in 2013 and 2017). This delay was associated with the process of IDEA being converted to an Infrastructure Project.

\section{Data analysis}

To evaluate the IDEA surveillance system we took a three-pronged approach including process observation, in-depth interviews and secondary data analysis. Interviewees included representatives from the three Western Australian government departments involved in the IDEA system, community representatives and researchers using a 20-item semi-structured questionnaire. The aim of the interview was to discuss the usefulness, simplicity, flexibility, acceptability, timeliness, data quality, representativeness and stability of the IDEA system through assessing and understanding responses of stakeholders. The questionnaire was administered face-to-face, took between 30 and $60 \mathrm{~min}$ and was recorded with participant's consent for further analysis. Some interviewees were not able to answer all questions depending on their level of involvement with the IDEA system. Thematic analysis according to the system attributes was completed.

Secondary data analysis was used to assess the data quality through determining the completeness of data. Cross-checking of individuals born between 1983 and 2014 from the mandatory Western Australian Registry of Developmental Anomalies-Cerebral Palsy database (WARDA-CP) who have ID to the IDEA system was undertaken. ID for the WARDA-CP database is ascertained through medical records and is updated when a child is 5 years of age. If there is no record of ID, the child's medical record will be checked again once they have started school. All confirmed cases from the IDEA system from 1983 to 2014 were included. Children from the WARDA-CP database were included in the analysis if they had a mild impairment (IQ or development quotient (DQ) 50-69), moderate impairment (IQ/DQ 35-49) or severe impairment $(\mathrm{IQ} / \mathrm{DQ}<35)$. Cross-tabulations were completed to determine the number of children from the WARDA-CP database that were not identified in the IDEA system. If there was a discrepancy between databases, further investigation to determine reasons for missing cases was completed.

\section{Ethics approval}

Written consent was provided and all data collected were anonymous. 


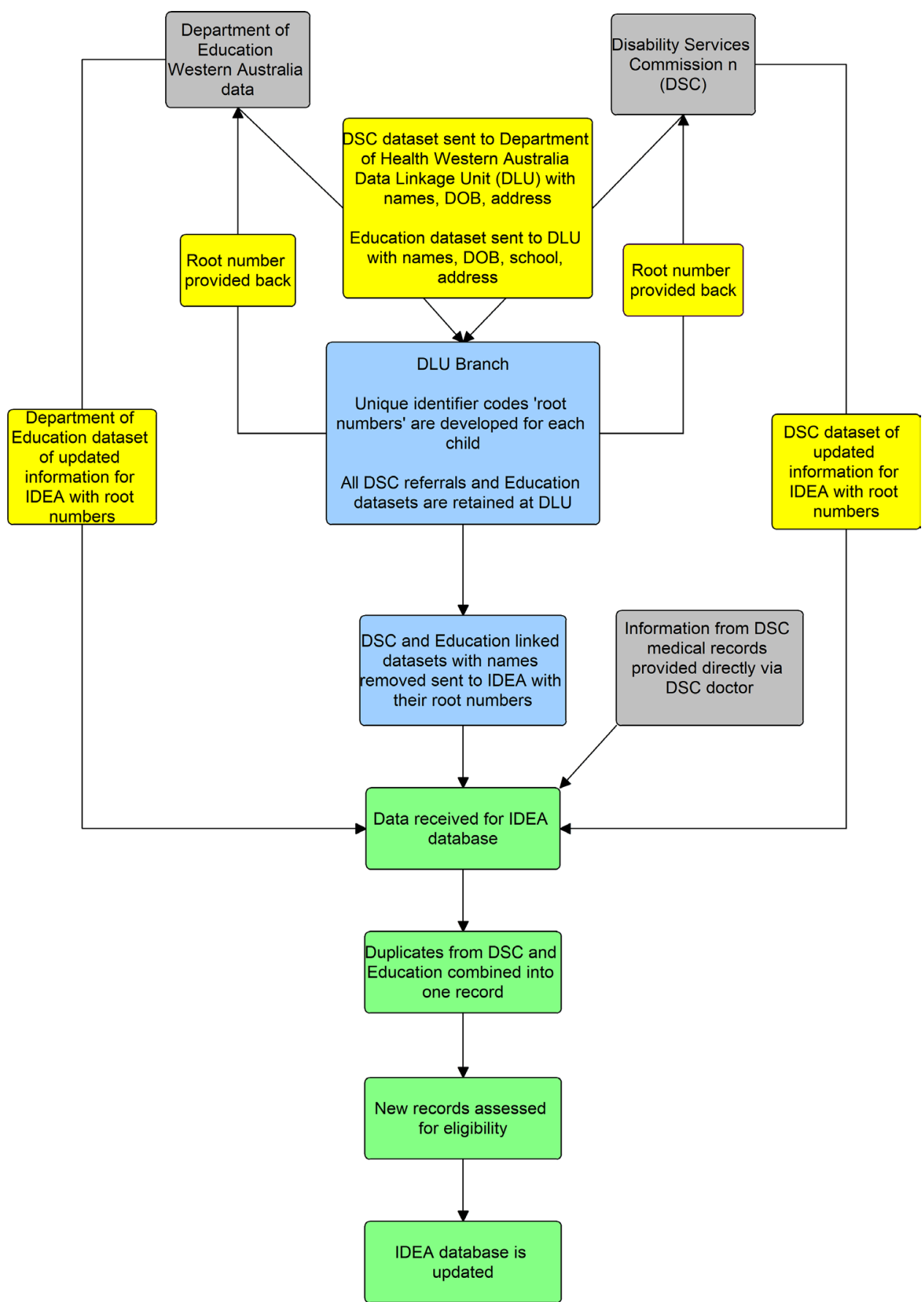

Figure 1 Flow diagram of process of data collection for IDEA surveillance system. Yellow identifies processes needed for confidentiality. Grey identifies where data are coming from. Blue identifies Department of Health Western Australia DLU involvement. Green identifies IDEA database final processes. The processes in yellow, grey and blue take 9 months to complete. The process outlined in green takes approximately 4 months to complete. DOB, date of birth; IDEA, Intellectual Disability Exploring Answers.

\section{RESULTS}

\section{Characteristics}

Eleven interviews were completed. Three individuals were solely involved in reporting, analysing and/or interpreting of ID surveillance data. Two contributed to the data either directly or in an advisory capacity. Six were involved in both of these roles. Interviewees had been involved with the IDEA system for 3-17 years and many of them contributed to the system in a number of different areas (table 1). Other roles that were identified included administrative support, reporting, communication and translation.

\section{Usefulness}

Reasons for the importance of identifying and collecting ID data included:

- Identifying prevalence and trends in ID. 
Table 1 Roles identified by interviewees within the IDEA surveillance system

\begin{tabular}{ll}
\hline Characteristics & Numbers (\%)* \\
\hline Analysing data & $8(73 \%)$ \\
\hline Reporting data & $8(73 \%)$ \\
\hline Interpreting data & $9(82 \%)$ \\
\hline Maintenance of data & $5(45 \%)$ \\
\hline Data quality & $7(64 \%)$ \\
Committee member & $4(36 \%)$ \\
\hline Data entry & $3(27 \%)$ \\
\hline Data linkage & $3(27 \%)$ \\
\hline Data extraction & $3(27 \%)$ \\
\hline Management of data & $6(55 \%)$ \\
Advocacy & $5(45 \%)$ \\
\hline
\end{tabular}

*The are multiple counts.

IDEA, Intellectual Disability Exploring Answers.

- Using data for prevention of ID and to understand causes of ID, and management of care services.

- Identifying subgroups such as comorbidity with mental illness, or child neglect for which ID is a very strong risk factor.

- Measuring and evaluating life outcomes for people with ID by being able to identify them as they move through the service system.

- Informing policy and practice particularly from a systems perspective for planning and resource allocation, particularly as people with ID are the largest single cohort of individuals receiving support through all disability services.

Concern about the stigma associated with identifying people as having an ID was expressed, although services, funding and resource allocation decisions are made as a result of these processes. Ensuring appropriate identification was considered an important part of the data collection process.

Interviewees thought the IDEA surveillance system had either met or partially met the overall aim of the IDEA system; to provide high-quality, complete and population-based information on ID in Western Australia. The IDEA surveillance system was considered to be an infrastructure which had provided a substantial amount of data to assess trends in the prevalence of ID, investigate health service use for people with ID, evaluate risks associated with having an ID and health and social determinants of ID. However, a major drawback in 2010 was the loss to the database of any information from the Western Australian Midwives database, which provides an individual's basic birth data (born in Western Australia, race, birth weight, etc). As a result, other than through separate ethically approved data linkage projects, many of which have been undertaken, it is now difficult to provide many routine statistics. The system was also considered to be missing subgroups of individuals such as the small number of people attending Catholic or independent schools for children born since 1992, individuals who were not receiving services from DSC or those not using the state education system. Additional data variables such as genetic information related to an individual's ID, comorbidities and in particular functional capacity, were commonly cited among interviewees as important information for IDEA. Interviewees agreed that evaluations of screening programme for prevention, early intervention or therapy programme for ID or genetic research into the causes and prevention of ID had not been possible because of lack of availability of data or, if available, the presence of ethical and other constraints to its linkage. Lastly, it was acknowledged that although professional knowledge had increased about ID, it was not known what impact this may have had on community awareness.

All interviewees had either used or read about the IDEA data in journal publications, annual reports, stakeholder reports, reports for consumers or the public, policy briefs, government reports, newsletters, minister reports, book chapters and conferences. There have been over 40 journal publications with approximately 740 citations and 70 conference presentations between 2004 and 2017 that have used IDEA surveillance data. Importantly, IDEA data have been widely used, cited and published in international literature including in international estimates of years lived with disabilities (2010) ${ }^{13}$ In addition, many of the 40 journal articles have investigated both the determinants of ID and associated outcomes like comorbidities and premature mortality. However, there was unanimous agreement that there needs to be more publications, particularly consumer and policy-driven, as well as regular biannual reports. Although there had been direct engagement with the DSC Director General through meetings every 3 months in 2013 facilitated with philanthropic funding to provide information on outcomes, it was considered by many interviewees that there had been little in the way of communicating results to the community and advocacy organisations. It was suggested knowing this information could be beneficial for community groups to advocate with and for families and individuals with ID.

\section{Simplicity}

There were conflicting responses when asked about the simplicity of the system. Respondents discussed the process for collecting data for the IDEA surveillance system inconsistently as simple, timely, complex or taking too long (figure 1). However, the process for collecting data is largely based on safeguarding privacy, therefore the 9 months it takes for the IDEA team to receive data were deemed by those who have worked with and in the Department of Health to be in line with current data linkage processes. The 4 months for integrating data received by government departments into the IDEA system were considered reasonable, especially as there is only one person working 0.5 full time equivalent (FTE). The process of providing ID data for research projects was 


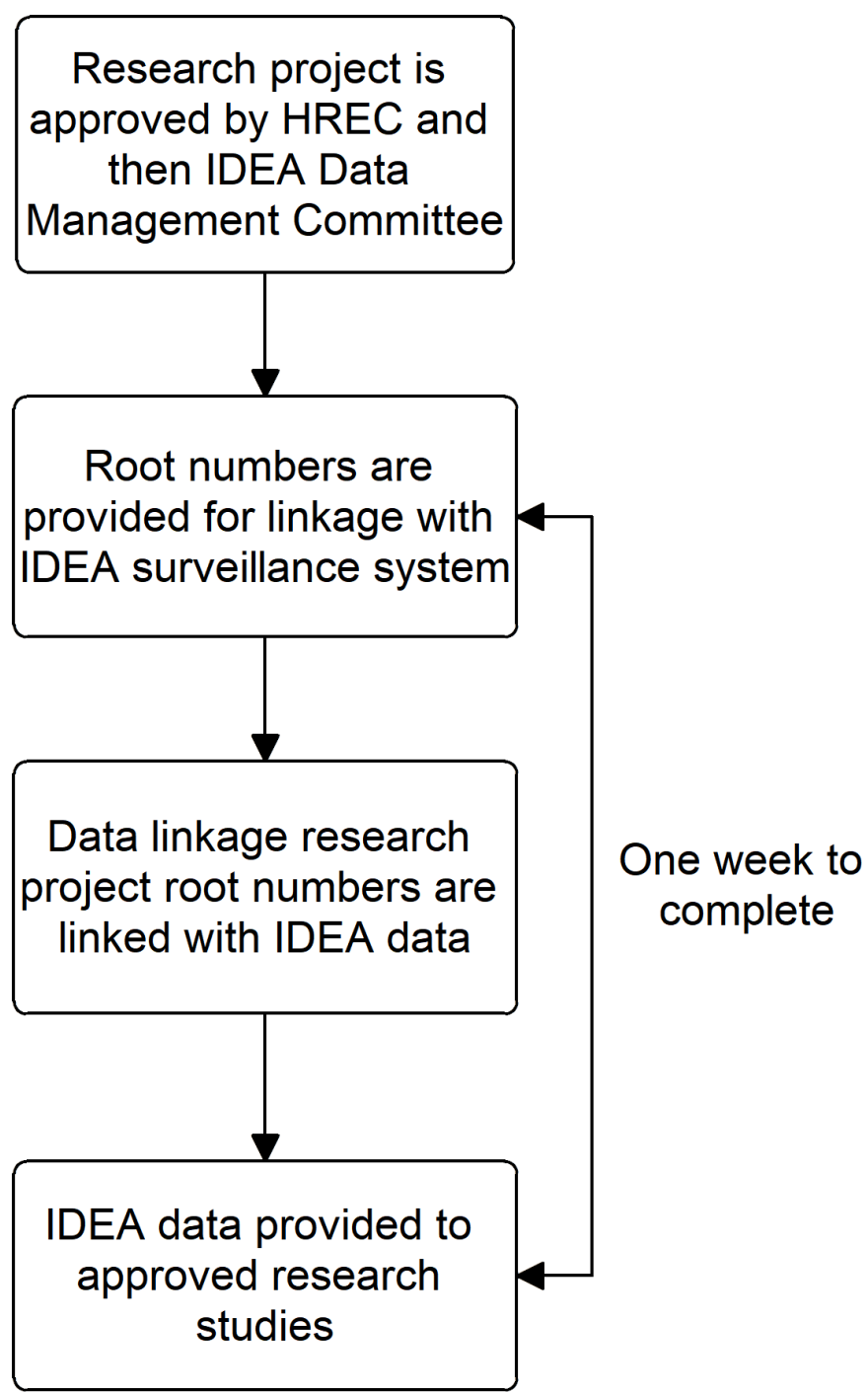

Figure 2 Internal data linkage process for IDEA surveillance data. HREC, Human Research Ethics Committee; IDEA, Intellectual Disability Exploring Answers.

also perceived as appropriate and completed in a timely manner (figure 2).

\section{Flexibility}

The IDEA system was relatively flexible to changes in personnel and case definitions. Personnel and process changes have occurred at all stages within the data process, with the exception of the Telethon Kids Institute (TKI) team. The TKI team has largely remained the same since the inception of the system in 2002. As a result, the process of data linkage and extraction from the larger dataset received from Department of Health to the TKI team has not been documented to date. Although having a consistent team has created a system that is flexible and stable, as part of good practice and sustainability, developing formalised documented processes would be valuable. Case definitions have also varied with changes in how the Department of Education have recorded ID. These changes have been recorded and the system adapted accordingly for data integrity.
Data quality

There was universal agreement that the system was not complete for ID in Western Australia with people attending Catholic or independent schools and individuals who were not receiving services from DSC likely to be missing from the system. In addition since mid-2014, individuals living in the Perth Hills region who were part of the National Disability Insurance Scheme pilot location had their data collected by the Australian Commonwealth government rather than the state-based government. ${ }^{14}$ Therefore, there will be no data available on newly registered individuals with ID from this location at the next IDEA update.

Data quality is the responsibility of the two departments that assess individuals for ID. Each department has its own assessments for ID, reasons for collecting ID and ways in which the information is used. Ensuring data quality across organisations and that individuals with ID are correctly identified was seen as important for all people involved in collecting and using data.

We also assessed the completeness for individuals in the IDEA surveillance system to a subgroup of individuals, cerebral palsy with ID, from the mandatory reporting surveillance system WARDA-CP. Overall, there were 10593 cases of ID in the IDEA system; 582 individuals were identified in the WARDA-CP surveillance system as having cerebral palsy and ID. Of those identified, 501 (86.1\%) were also in the IDEA system and $81(13.9 \%)$ were not. In total, $0.7 \%$ of cases $(81 / 10674)$ with ID were not identified in the IDEA system. Potential reasons for the discrepancies between the two sources were children who had died prior to school entry may not be identified in IDEA $(n=8)$ and that WARDA-CP may be including cases with probable or borderline ID who would not be eligible for IDEA. There were little differences in Indigenous status, sex and place of residence for cases not identified in the IDEA system (table 2).

\section{Acceptability}

There are four organisations (TKI, Department of Health Western Australia, DSC, Department of Education) within Western Australia that voluntarily participate in the IDEA surveillance system. Unlike other surveillance systems, there are no mandatory requirements for case notification and therefore no onus on clinicians and other public health practitioners to participate. The two departments which supply data for the IDEA system do so voluntarily and deem the collection of data to be important. Memoranda of understanding have been signed by DSC and Education with the Department of Health for the release of data. In addition, there is an agreement between TKI and Department of Education outlining the provision of education data to IDEA and a Grant Agreement between TKI and DSC.

\section{Representativeness}

ID data within the IDEA surveillance system are dependent on individuals being referred (by clinicians, 
Table 2 Comparison of IDEA and WARDA-CP surveillance system data, 1982-2014

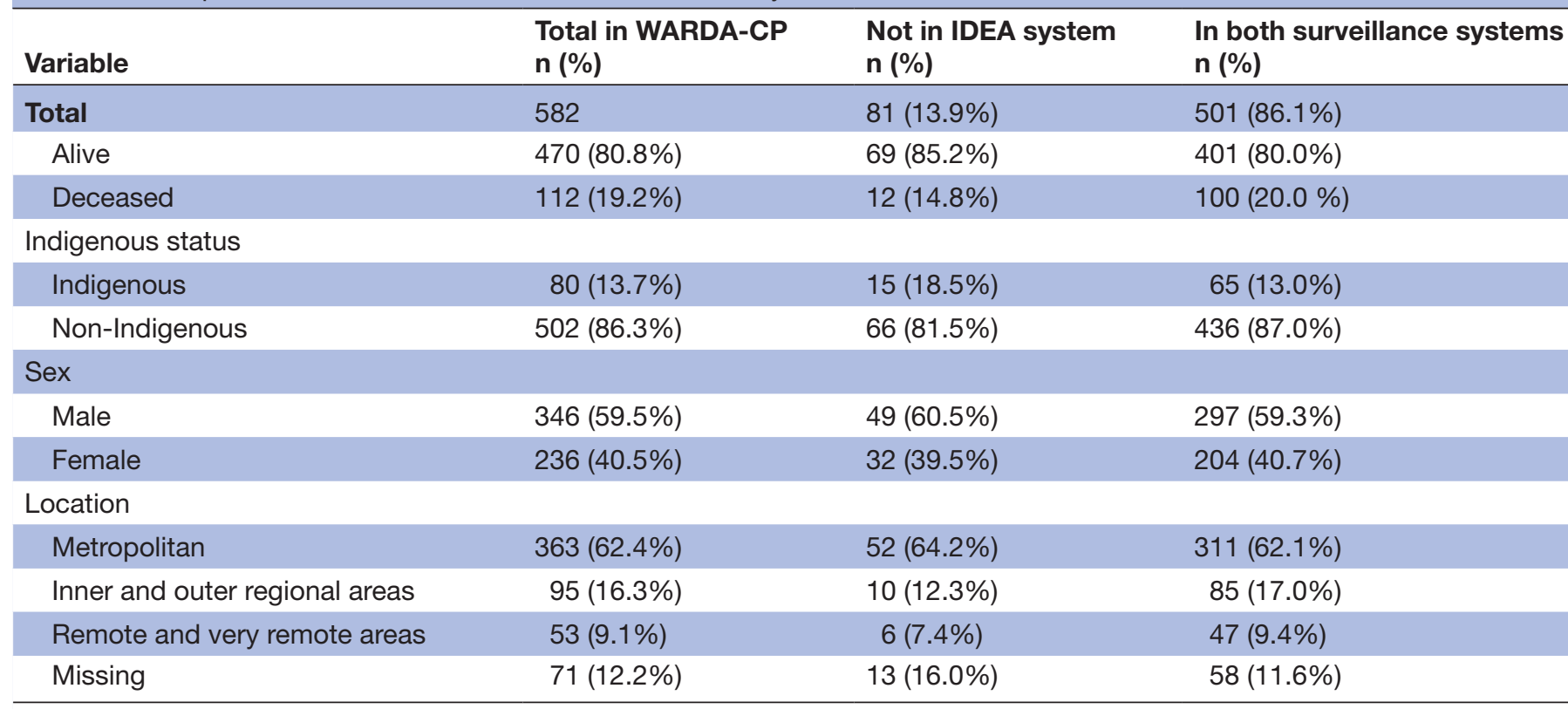

IDEA, Intellectual Disability Exploring Answers; WARDA-CP, Western Australian Registry of Developmental Anomalies-Cerebral Palsy database.

psychologists, allied health, teachers or parents) for services and/or being identified through the public education system. Since the IDEA system does not have mandatory notifications, it is not surprising that there are certain subgroups of individuals who may not be represented. Despite this, there is no other equivalent system elsewhere in Australia and these data have been used as a key data source for ID national estimates. ${ }^{15}$ As a result, the epidemiology findings are considered generalisable to the larger Australian population.

\section{Timeliness}

Overall, the timeliness of the data was considered to be appropriate including the 2-year period between data extractions. The initial 9 months for the data linkage process has previously been delayed through new staff having to extract the data from the two departments, resource limitations and priority delays within the departments. These barriers have resulted in delays at all stages of the 9-month data extraction. It was also discussed that some of these time delays were the result of ensuring confidentiality, however, this is an important component of the system. Alternatively, it was mentioned that if individuals, organisations and policymakers valued the data then more frequent data extractions could occur.

\section{Stability}

Despite being a non-mandatory surveillance system, data have been regularly provided by departments and there has been ongoing funding negotiated. The funding provided has allowed for a 0.5 FTE position which supports personnel and operating costs. However, in-kind support from the TKI disability team has also supported these activities and the day-to-day administrative tasks.
The limited funding for the database has also restricted the amount of work that can be achieved within the IDEA system. Additional activities could include engaging with stakeholders, translation and communication of findings, use of IDEA data for supporting policy decisions and priority setting. It was estimated by those working directly with the system that 1.5 FTE would be enough to complete the technical requirements of the IDEA surveillance system and be able to complete the additional tasks outlined.

\section{DISCUSSION}

The IDEA system is the only Australian population-based ID surveillance system and one of few internationally. ${ }^{16-18}$ Since 2002, the IDEA system has been successfully funded and maintained by long-term collaborations with two Western Australian departments. This has provided an infrastructure to understand prevalence rates and trends over time for ID, inform resource allocation, identify those at risk of negligence or other adverse events, identify risk and protective factors associated with ID and inform larger international studies on the global burden of disability. ${ }^{13} 19-22$ Overall, the IDEA system was considered to be flexible, simple, acceptable, representative, timely and stable. However, components within these attributes such as insufficient engagement with stakeholders and community, lack of opportunities for translation and ensuring there is a workforce to deliver these initiatives could be improved.

Although many participants thought that subgroups were missing within the IDEA system, this is likely to be a very small percentage of the population. Case 
ascertainment using two resources is high with previous research showing that between 1983 and 2003 only 50\% of cases were ascertained through the DSC Services, with the remaining $50 \%$ from the Department of Education. ${ }^{23}$ In addition, when considering the quality and quantity of services provided, as seen in Western Australia, using administrative data sources results in high ascertainment of cases and therefore sound reporting of prevalence rates. ${ }^{24}$ When comparing whether the WARDA-CP system had any additional cases not in the IDEA system, there was a small percentage of cases missing. This equated to $<1 \%$ of total cases in the IDEA system and reflects the highquality data source. The IDEA system provides coverage of ID considerably superior to that from other administrative datasets such as the Western Australia Hospital Morbidity Data System. ${ }^{25}$ Overall, the completeness of the IDEA system was high when compared with potential missing population data.

Due to the IDEA system's data linkage capabilities, data from Western Australian data collections including health, justice and child protection can be linked to determine important and complex associations both cross-sectionally and longitudinally for people living with intellectual disabilities. High-quality linked administrative data collections for determining adverse outcomes for people living with ID have been used internationally. ${ }^{26-29}$ In the UK, an inquiry found people living with an ID were more likely to experience avoidable deaths. ${ }^{29}$ Recommendations from this enquiry were to develop a central registration system for people with learning disabilities to ensure they receive appropriate care. Other country examples of using linked administrative data collections to determine the service utilisation and health disparities for people living with ID and those without include Canada, ${ }^{27}{ }^{30}$ Scotland ${ }^{2631}$ and the USA. ${ }^{28} 32$

\section{Current and future impact of ID data}

In Australia, the methods for capturing and reporting on ID are some of the best in the world. Aside from Canada, the Western Australian IDEA systems infrastructure and data linkage capabilities have resulted in one of the largest repositories of data. ${ }^{33}$ Data have been used, but not limited to identifying important health disparities, psychiatric comorbidities and health service patterns, including the last year of life among people living with an ID..$^{2034}$ IDEA data have also contributed to prevalence patterns, identifying increased mortality risk for children at different ages and the burden of ID globally. ${ }^{13} 1922$ Box 1 provides a summary of important policy-relevant and programme-relevant implications and findings for both Australia and internationally.

More recently, research published using IDEA data has shown that children with an ID were at higher risk of child maltreatment allegations compared with children without a disability. ${ }^{1}$ These results have been reported by researchers from the TKI to the Australian Royal Commission into Institutional Responses to Child Sexual Abuse in regards to identifying the prevalence of child
Box 1 Examples of policy-relevant and programmerelevant findings for people living with an intellectual disability

\section{Antenatal care}

- Improved management of women with diabetes, epilepsy and/or anaemia during the antenatal period to reduce the risk of having a child with intellectual disabilities. ${ }^{37}$

- Importance of monitoring maternal health due to poor fetal growth increasing the risk of intellectual disability. ${ }^{35}$

- Health promotion and public health campaigns to prevent the use of alcohol during pregnancy. ${ }^{38}$

\section{Service delivery}

- Children with intellectual disability are also more likely to have birth defects resulting in increased health and social supports for children and additional services for families. ${ }^{39}$

- The need for additional services and support for families in areas of social disadvantage who are at greater risk of having child with intellectual disability. ${ }^{35}$

- Improved access, quality and coordination is needed for individuals with intellectual disability as they are more likely to experience potentially preventable conditions at the end of their lives. ${ }^{34}$

sexual abuse among children with disabilities. ${ }^{36}$ In addition, these data were also used to advocate and successfully implement a disability indicator within the Western Australia Department of Communities-Child Protection and Family Support and the Australian National Child Protection Minimum Dataset in order to improve the detection and management of children with an ID who have experienced maltreatment. ${ }^{36}$ This indicator will also assist in the development of maltreatment prevention strategies for children with an ID. Examples such as these demonstrate how high-quality data are fundamental in dealing with the challenging health and social issues of people living with IDs.

Although there has been substantial work completed in the local context for people living with an ID, there is still more that the IDEA system can contribute to internationally. There are still large gaps in the evidence including understanding mortality rates among those with IDs including using consistent methodology and cases definition, differentiating between specific syndromes and creating pooled mortality estimates from different countries through virtual minimum datasets to determine the global mortality rate attributed to ID. The IDEA system is well set up to contribute to these important local and international research priorities.

\section{Lessons learnt}

There are two main indicators that have resulted in the success of the IDEA system. The first is the vision and leadership of data custodians and those who saw the immense value of having a population-based data collection for ID. These individuals have made substantial contributions to research and policy translation of people living with an ID in Western Australia. The second is Western Australia's high-quality data linkage system, which helped them make 
their vision become a reality. Although other governments may not be able to achieve this level of population-based data linkage, other alternative data collections exist such as national surveys, registries and hospital data. These alternatives have been used for determining prevalence of ID in low-income and middle-income countries, which have also made important contributions to ID data. ${ }^{17}$

\section{Recommendations}

The IDEA surveillance system has provided important clinical data on the health and social needs of people living with an ID. Despite this there are a number of areas that the IDEA team could undertake to strengthen the system. Based on this evaluation we recommend the following:

1. Discussion and engagement with the IDEA Advisory Group on the collection of ID given the changes in data ownership.

2. The IDEA team has been involved in the system since its initiation in 2002. As a result, there have been few protocols developed for how data are linked, extracted and maintained. It is recommended that internal protocols are developed for future personnel working on the system. An additional 1 FTE is also recommended to support additional activities proposed in these recommendations.

3. Active engagement with community and relevant stakeholders including disability organisations, policy makers, researchers and service organisations is sorely needed to promote awareness of current research and to determine priority setting for future research. This can be achieved through the development of communication and translation strategies as well as priority setting workshops.

4. Currently, the IDEA team uses the Heber classification for the level of disability. This is an outdated system with other classification systems more up to date with current practice. Determining whether there are other classification systems that could be used and if the data could be moved to this system would be beneficial.

5. An additional variable for functional ability was considered to be important for informing current practice. Enhanced surveillance on a subgroup of individuals could be considered. To determine whether these data are important and if so what data would be included should occur in consultation with stakeholders.

6. The Advisory Group should consider meeting annually again. This increased level of active engagement and strategic planning could influence the current activities of IDEA and inform future directions. Leadership is needed and the Advisory Group are well placed to take on this role.

\section{CONCLUSION}

The IDEA surveillance system provides crucial data about people living with ID. However, there remains significant challenges in the future of the IDEA system given recent funding and service delivery changes within Australia. Changes to engagement with the community and stakeholders could play an essential role in the sustainability of the IDEA system through advocacy for its continuation. Enhanced surveillance for functional capacity could also strengthen the system and provide important information for people living with ID and their families. The IDEA surveillance system is one of the few international ongoing data collections of ID. Discontinuing data collection and evaluation for this vulnerable population would be a disservice to society. Implementation of these recommendations will provide ways for the IDEA system to remain a successful source of important data for people living with an ID.

Acknowledgements The authors would like to thank the stakeholders in providing their thoughts on the IDEA system. The authors would also like to thank DSC and Department of Education for their ongoing support of the IDEA database.

Contributors Conceived and designed the experiments: NAS, JB, HL, AR, DMcA. Performed the experiments: NAS, JB. Analysed the data: NAS. Wrote and provided intellectual input into the paper: NAS JB, HL, AR, KME, DMcA. All authors approved the final version. All authors are accountable for all aspects of the paper.

Funding This research is supported by an Australian Government Research Training Program (RTP) Scholarship.

Disclaimer The funders had no role in the design of the study and collection, analysis and interpretation of data and in writing the manuscript.

Competing interests $\mathrm{JB}$ is employed to work on the IDEA system. $\mathrm{HL}$ is the data custodian of the IDEA system.

Patient consent for publication Not required.

Ethics approval This study was approved by the Department of Health Western Australia Human Research Ethics Committee (2014/24), The University of Western Australia (RA/4/20/4168) and The Australian National University (2017/567).

Provenance and peer review Not commissioned; externally peer reviewed.

Data availability statement Data are available through ethical approval from the relevant ethics committees in collaboration with the authors.

Open access This is an open access article distributed in accordance with the Creative Commons Attribution Non Commercial (CC BY-NC 4.0) license, which permits others to distribute, remix, adapt, build upon this work non-commercially, and license their derivative works on different terms, provided the original work is properly cited, appropriate credit is given, any changes made indicated, and the use is non-commercial. See: http://creativecommons.org/licenses/by-nc/4.0/.

ORCID iDs

Natalie A Strobel http://orcid.org/0000-0002-2962-5704

Helen Leonard http://orcid.org/0000-0001-6405-5834

Alice Richardson http://orcid.org/0000-0001-7084-1524

\section{REFERENCES}

1 Maclean MJ, Sims S, Bower C, et al. Maltreatment risk among children with disabilities. Pediatrics 2017;139:e20161817.

2 Cooper S-A, McLean G, Guthrie B, et al. Multiple physical and mental health comorbidity in adults with intellectual disabilities: population-based cross-sectional analysis. BMC Fam Pract 2015;16:110.

3 Emerson E. Mothers of children and adolescents with intellectual disability: social and economic situation, mental health status, and the self-assessed social and psychological impact of the child's difficulties. J Intellect Disabil Res 2003;47:385-99.

4 Evans E, Howlett S, Kremser T, et al. Service development for intellectual disability mental health: a human rights approach. $J$ Intellect Disabil Res 2012;56:1098-109.

5 Bittles AH, Petterson BA, Sullivan SG, et al. The influence of intellectual disability on life expectancy. J Gerontol A Biol Sci Med Sci 2002;57:M470-M472. 
6 Centers for Disease Control and Prevention. Metropolitan Atlanta developmental disabilities surveillance program (MADDSP), 2015 Available: https://www.cdc.gov/ncbddd/developmentaldisabilities/ MADDSP.html [Accessed 17 Jan 2018]

7 Ouellette-Kuntz H, Martin L, McKenzie K, et al. Chapter Six - A Review of Health Surveillance in Older Adults with Intellectual and Developmental Disabilities. In: International review of research in developmental disabilities. Academic Press, 2015: 151-94.

8 Krahn G, Fox MH, Campbell VA, et al. Developing a health surveillance system for people with intellectual disabilities in the United States. J Policy Pract Intellect Disabil 2010;7:155-66.

9 Leonard H, Glasson E, Bebbington A. Chapter Eight - Application of Population-Based Linked Data to the Study of Intellectual Disability and Autism. In: Urbano RC, ed. International review of research in developmental disabilities. Academic Press, 2013: 281-327.

10 Leonard H, Petterson B, Bourke J. Inaugural report of the IDEA database - intellectual disability in Western Australia. Perth, WA Telethon Institute for Child Health Research; 2004.

11 Centers for Disease Control and Prevention. Updated guidelines for evaluating public health surveillance systems: recommendations from the guidelines working group: MMWR, 2001; 50(No. RR-13); 2001: 1-51.

12 Heber R. A manual on terminology and classification in mental retardation. Am J Ment Defic 1959;64:1-111.

13 Vos T, Flaxman AD, Naghavi M, et al. Years lived with disability (YLDs) for 1160 sequelae of 289 diseases and injuries 1990-2010: a systematic analysis for the global burden of disease study 2010 . The Lancet 2012;380:2163-96.

14 National Disability Insurance Agency. Annual report: 2014-2015. Canberra National Disability Insurance Agency; 2015.

15 AlHW. Australian burden of disease 2011: methods and supplementary material. Australian burden of disease study series No. 5. cat. No. BOD 6. Canberra AIHW; 2016.

16 CDC. Metropolitan Atlanta developmental disabilities surveillance program (MADDSP), 2017. Available: https://www.cdc.gov/ncbddd/ developmentaldisabilities/MADDSP.html [Accessed 5 Apr 2018].

17 Maulik PK, Mascarenhas MN, Mathers CD, et al. Prevalence of intellectual disability: a meta-analysis of population-based studies. Res Dev Disabil 2011;32:419-36.

18 Urbano RC. Chapter Nine -Large-Scale Datasets Referenced in Volume 45 of the International Review of Research in Developmental Disabilities. In: Urbano RC, ed. International review of research in developmental disabilities. Academic Press, 2013: 329-42.

19 Bourke J, de Klerk N, Smith T, et al. Population-Based prevalence of intellectual disability and autism spectrum disorders in Western Australia: a comparison with previous estimates. Medicine 2016;95:e3737

20 Morgan VA, Leonard H, Bourke J, et al. Intellectual disability co-occurring with schizophrenia and other psychiatric illness: population-based study. Br J Psychiatry 2008;193:364-72.

21 Fitzgerald $\mathrm{P}$, Leonard $\mathrm{H}$, Pikora TJ, et al. Hospital admissions in children with Down syndrome: experience of a population-based cohort followed from birth. PLoS One 2013;8:e70401.

22 Bourke J, Nembhard WN, Wong K, et al. Twenty-Five year survival of children with intellectual disability in Western Australia. J Pediatr 2017;188:232-9.
23 Petterson B, Leonard H, Bourke J, et al. IDEA (intellectual disability exploring answers): a population-based database for intellectual disability in Western Australia. Ann Hum Biol 2005;32:237-43.

24 Yeargin-Allsopp M, Murphy CC, Oakley GP, et al. A multiple-source method for studying the prevalence of developmental disabilities in children: the metropolitan Atlanta developmental disabilities study. Pediatrics 1992:89:624-30.

25 Bourke J, Wong K, Leonard $\mathrm{H}$. Validation of intellectual disability coding through Hospital morbidity records using an intellectual disability population-based database in Western Australia. BMJ Open 2018;8:e019113.

26 Cooper S-A, Hughes-McCormack L, Greenlaw N, et al. Management and prevalence of long-term conditions in primary health care for adults with intellectual disabilities compared with the general population: a population-based cohort study. J Appl Res Intellect Disabil 2018;31 Suppl 1:68-81.

27 Balogh RS, Lake JK, Lin E, et al. Disparities in diabetes prevalence and preventable hospitalizations in people with intellectual and developmental disability: a population-based study. Diabet Med 2015;32:235-42

28 Chapman DA, Scott KG, Stanton-Chapman TL. Public health approach to the study of mental retardation. Am J Ment Retard 2008;113:102-16.

29 Heslop P, Blair PS, Fleming P, et al. The Confidential inquiry into premature deaths of people with intellectual disabilities in the UK: a population-based study. The Lancet 2014;383:889-95.

30 Balogh R, Brownell M, Ouellette-Kuntz H, et al. Hospitalisation rates for ambulatory care sensitive conditions for persons with and without an intellectual disability--a population perspective. J Intellect Disabil Res 2010;54:820-32.

31 Mackay DF, Smith GCS, Cooper S-A, et al. Month of conception and learning disabilities: a record-linkage study of 801,592 children. Am J Epidemiol 2016;184:485-93.

32 Chapman DA, Scott KG, Mason CA. Early risk factors for mental retardation: role of maternal age and maternal education. Am J Mental Retard 2002;107:46-59.

33 Balough R, Leonard H, Bourke J, et al. Data linkage: Canadian and Australian perspectives on a valuable methodology for intellectual and developmental disability research. J Intellect Dev Disabil.

34 Brameld K, Spilsbury K, Rosenwax L, et al. Use of health services in the last year of life and cause of death in people with intellectual disability: a retrospective matched cohort study. BMJ Open 2018;8:e020268.

35 Leonard H, Glasson E, Nassar N, et al. Autism and intellectual disability are differentially related to sociodemographic background at birth. PLoS One 2011;6:e17875.

36 O'Donnell M. IDEA system and child protection (personal communication to N.A. Strobel); 2019.

37 Langridge AT, Glasson EJ, Nassar N, et al. Maternal conditions and perinatal characteristics associated with autism spectrum disorder and intellectual disability. PLoS One 2013;8:e50963.

38 O'Leary C, Leonard H, Bourke J, et al. Intellectual disability: population-based estimates of the proportion attributable to maternal alcohol use disorder during pregnancy. Dev Med Child Neurol 2013;55:271-7.

39 Petterson B, Bourke J, Leonard H, et al. Co-Occurrence of birth defects and intellectual disability. Paediatr Perinat Epidemiol 2007;21:65-75 